\title{
Ethical Considerations in Clinical Biochemistry and Laboratory Medicine: A Discussion Based on 'The Belmont Report'
}

\author{
Miliva Mozaffor ${ }^{1}$, Mariya Tabassum², Mohammad Tipu Sultan ${ }^{3}$, Shamima Parvin ${ }^{4}$ \\ 1. Clinician Researcher, Biomedical Research Foundation (BRF), Dhaka \& Laboratory Consultant and Head, \\ Department of Biochemistry, Ashiyan Medical College Hospital, Dhaka-1229, Bangladesh. \\ Email: miliva17@yahoo.com (corresponding author) \\ 2. Assistant Professor, Department of Biochemistry, Pabna Medical College, Pabna-6600, Bangladesh. \\ 3. Postgraduate student, Department of Forensic Medicine \& Toxicology, Dhaka Medical College, Dhaka-1000, \\ Bangladesh. \\ 4. Professor and Head, Department of Biochemistry \& Vice-Principal, Mugda Medical College, Dhaka-1214, \\ Bangladesh.
}

\begin{abstract}
With technical sophistication and innovation in the field of medical science, a considerable proportion of medical diagnosis now rely on laboratory analyses, which emphasises the crucial role of laboratory physicians in patient care. Sustaining high ethical standards remains crucial in both clinical biochemistry and laboratory medicine, and several ethical dilemmas are faced by laboratory physicians in day-to-day practice. In a low-resource country like Bangladesh, formal ethics education or ethical framework in laboratory practice is still absent; ethics has not received that much attention it this field. This paper has considered ethical issues encountered during the daily routine work of laboratory physicians and specially focused on the ethical issues encountered during the pre-analytical, analytical and post-analytical phases of laboratory medicine practice and discuss those issues in light of 'The Belmont Report' (1978) perspective. It is not intended to be a comprehensive one, rather it aims to complement existing guidelines and documents that are available in some institutions and to offer a framework for addressing ethical issues encountered in the practice of clinical biochemistry and laboratory medicine in Bangladesh.
\end{abstract}

Key words: The Belmont Report, ethics, clinical biochemistry, laboratory medicine, Bangladesh.

Introduction: With technical sophistication and innovation in the field of medical science, a considerable proportion of medical diagnosis now rely on laboratory analyses, which emphasises the crucial role of laboratory physicians in patient care $^{1}$. In laboratory medicine, there is usually no direct or minimal contact with patients; however, the laboratory physicians' first and foremost duty is to act in the best interests of the patient who is often "just a number" (as coded) ${ }^{2}$. Although personal knowledge of the patient is often lacking, the laboratory physician does have intimate knowledge of at least a part of the patient - "the labelled specimen" - and an unusual but traditional three-way contract is made between clinician, laboratory physician and patient ${ }^{2,3}$. This is the case in particular where a life-altering event may occur on the basis of the definitive decision of a laboratory physician and the act of faith with which this is accepted by the patients $^{1-3}$.

The evolution of medical ethics over the years is well documented and evolved 
through the Nuremberg Code from $1947^{4}$, the Declaration of Geneva from September 1948, with its continual amendment until October $2017^{5}$, the Declaration of Helsinki from June 1964, with its continual amendment until October $2013^{6}$ and 'The Belmont Report' from $1978^{7}$. While many of these documents focus on medical research, concepts in the Declaration of Geneva and The Belmont Report are also applicable to the practice of clinical medicine to date. Moreover, 'The Belmont Report' remains one of the key milestones concerning ethics in biomedical research. Created in 1978 by the National Commission for the Protection of Human Subjects of Biomedical and Behavioral Research of the United States, it outlines ethical principles and guidelines for the protection of human subjects. It identifies three core principles; those are as follows: ${ }^{7}$

a) Respect for persons: To ensure autonomy of the subjects/patients as well as to protect autonomy of those with diminished capacity to consent and make decision by themselves.

b) Beneficence: Acting in the best interests of patients or study subjects as well as maximize benefits and minimize harm. It is also termed as non-maleficence.

c) Justice: Moral obligation to treat all the patients equally disregarding age, sex, and race, and to ensure fair allocation of resources, e.g. treatment facilities and medications/vaccines, what is rightly due in terms of benefits, risks and cost.

As practicing physicians in the field, we have felt that sustaining high ethical standards remains crucial in both clinical biochemistry and laboratory medicine, and several ethical dilemmas are faced by laboratory physicians in day-to-day practice ${ }^{2}$. The importance of ethics in clinical biochemistry and laboratory medicine can never be ignored; however, there is variability in ethics education worldwide, focusing on the ethics in the laboratory procedure and practice. In a lowresource country like Bangladesh, formal ethics education or ethical framework in laboratory is still absent; ethics has not received that much attention it this field. As we have stated earlier, the three basic principles of 'The Belmont Report' can be applied to both research and clinical settings. In this paper, these three principles will mostly be highlighted to clarify the ethics in clinical biochemistry and laboratory medicine. This paper aims to focus on the ethical issues encountered during daily routine work of laboratory physicians and will consider the pre-analytical, analytical and post-analytical phases in laboratory medicine and discuss those in 'The Belmont Report' perspective.

\section{Ethical Issues in the Pre-analytical Phase:}

Usually it is assumed that the referring clinician has ordered the appropriate tests to support his/her provisional diagnosis, not for any financial gain, discussed the risks and benefits of the tests to the patients, referred testing to an appropriate laboratory (one that is that is properly certified and approved by the corresponding authority and in which the referring individual has no financial interest) and practiced those three ethical principles to the best of his/her ability. The maintenance of ethical standards in the pre-analytical phase is the collaborative responsibility of the laboratory, the health care provider, 
researcher, phlebotomist, nurse, or whoever collects the specimen $^{8}$. In pre-analytical phase, their roles include proper identification of the patient, collection of the appropriate sample using the appropriate technique, accurate identification and labeling of the sample so that the right tests are performed, and proper handling of the specimen until testing is performed ${ }^{8,9}$. In this stage, the application of those three principles is as follows:

a) Respect for persons: Consent should be obtained prior to sample collection, preferably a written one, and this consent should be informed (the patient knows what testing is being performed and why ${ }^{10-12}$. However, the consent may be implied when a patient provides a requisition from the clinician and willingly sits in a collection chair and allows a sample to be taken. However, implied consent is nuanced as a patient who is sitting to have blood collected may have been told what to do and may not actually understand that they had a choice ${ }^{8}$. Informed consent may pose an ethical problem if the patient is incompetent to make any decision due to age, mental status, or critical illness $^{10-12}$. However, who may be allowed to give consent on behalf of the patient may vary among regions, may be influenced by different cultural practices ${ }^{12}$. Besides, the patient's right to refuse to be tested or the methods of collection of samples, should be respected ${ }^{7}$. However, there are certain situations in which patient autonomy is not absolute. For example, a patient may be deemed incompetent to make a decision about his/her health, as when the patient is unconscious, mentally ill, or under the influence of $\operatorname{drugs}^{11,12}$. Children are generally considered as incompetent in decision-making for themselves until and unless they are legally emancipated from their parents; however, the status of children and adolescents under 18 years of age remains an area of controversy and is viewed differently in different parts of the world ${ }^{12}$. There are cases of compulsory testing in certain groups such as intravenous drug users and prisoners. In these exceptional cases, healthcare professionals have an obligation to consult the guidelines provided by the institution in which they practice, and they must weigh the risks of loss of a patient's autonomy versus the benefits of the testing ${ }^{11}$. Confidential information about patient demographics, the visit of a patient to a testing facility, which tests were ordered, and the reasons for those tests, should be given only to appropriate persons ${ }^{8,9,13}$. Confidentiality is a must at every step of the process - specimen transportation, data entry and report delivery ${ }^{13}$.

b) Beneficence: All tests should benefit the patient based on the best medical evidence. In addition, sample collection should not cause harm. Examples of harm in the preanalytical phase include infection or pain from the collection process (e.g. inadvertent puncture of an artery and other adverse events). Therefore, standard operating procedures and trained personnel should be in place ${ }^{8,9}$. Besides, the collection procedure should be carried out using universal precautions to protect both the patient and healthcare worker. Additional specimens shall not be collected for research procedures without 
informed consent from the patient and approval from the appropriate ethics board ${ }^{14}$. c) Justice: The clinical laboratory should, as far as it is able, provide access to a wide variety of laboratory tests at reasonable cost. There should be no preference given to individuals to facilitate or expedite the collection process at the expense of other patients $^{8-10}$.

Ethical Issues in the Analytical Phase: Confidentiality, quality assurance and competence are vital for all laboratories and settings. In the analytical phase, confidentiality may be maintained through automation that uses automated bar code readers, automated analysis, and autoverification, as the patient names are deemed by $\operatorname{codes}^{15}$. Nevertheless, challenges of ensuring confidentiality are often greater in small laboratories with low-resources that perform manual testing and in operations that conduct near-patient (point-of-care) testing. Now we look at those three principles:

a) Respect for persons: Patient have the right to decline to have their specimens analyzed even after the specimens have been collected and processed $^{8,9}$. Confidentially should be respected and maintained ${ }^{13,15}$. However, in point-of-care settings, it is really difficult because testing is often conducted in a common room with access by trained and non-trained personnel ${ }^{8}$.

b) Beneficence: The aim of the laboratory in the analytical phase is to provide the best possible analytical result. This is achieved through good laboratory practice and maintenance of professional standards. Good laboratory practice should involve the establishment of a rigorous quality assurance program encompassing quality control testing, proficiency testing and laboratory accreditation ${ }^{8,9,15}$. The maxim "a wrong result is worse than no result" is a guiding principle in this regard $8,15,16$. Good laboratory practice includes refusal to analyze or report a result when there is evidence of poor sample integrity, incorrect or poor labeling or other deficiencies that may compromise the test result ${ }^{8,9,17}$. Laboratories should maintain proper certification and only qualified, properly trained personnel should perform point-ofcare testing ${ }^{2,8}$.

c) Justice: Discrimination in the analysis of patient samples based on gender, age or racial origin is an injustice ${ }^{17}$. Laboratories should develop appropriate operating procedures for each type of testing. It is expected that all specimens are analyzed accurately and in a timely manner ${ }^{8,9,17}$.

\section{Ethical Issues in the Post Analytical Phase:}

The post analytical phase includes reporting and interpretation of results, residual specimen storage, and access to data. Laboratories should have a policy for specimen storage and data protection ${ }^{8,9}$. Archiving of results in either electronic or hard copy format is an important aspect of good laboratory practice ${ }^{15}$. Now we will discuss those three principles here:

a) Respect for persons: There are substantial differences in the world regarding the confidentiality of results. In some areas, the patient and the referring clinician are the sole legitimate recipients of laboratory data ${ }^{11}$. Exceptions are made if the patient is a juvenile or is incapable of receiving or understanding laboratory results. However, 
in other areas, the patient's family is regarded as legitimate recipients of a patient's laboratory results ${ }^{12}$. In addition, the patient should be allowed to give consent for access by others (such as family members) as required $^{12,13}$. Respect for local customs as to legitimate recipients of laboratory data should be taken into account as laboratories develop a policy on dissemination of results $^{13}$. In some areas, there may be exceptions regarding who may access results; access is affected by legal requirements and government regulations, especially in forensic cases ${ }^{12,13}$. Patients have a reasonable expectation that their samples will be used solely for the laboratory testing requested by the clinician. Individuals have the right to decide when and if their records or specimens shall be used outside the normal medical care to which they have consented ${ }^{12,14}$. Further testing of residual samples (except for method validation or in cases where samples are completely anonymized) should be approved by a local ethics committee or board, and patient consent may be required ${ }^{14}$. b) Beneficence: Misinterpretation of results can lead to patient harm; to minimize this harm, only qualified personnel should interpret reports ${ }^{15,16}$. It is expected that results should include an appropriate name for the test performed, an appropriate reference interval, which may be age and gender specific, the unit of measurement and, when possible, a designation that the test is within or above the reference interval. Timely access to results is important; withholding of results because of non-payment might lead to harming the patient especially in emergency situations ${ }^{17}$. Delays in reporting should be avoided. It is very crucial that ordering clinicians should be notified of errors as soon as those are identified, and test results should be corrected as soon as possible ${ }^{16}$.

c) Justice: The reporting of results should be consistent for all patients. Rapid reporting may be required for some results, such as for "critical" and "significant-risk" results; ${ }^{8}$ however, the rules for rapid reporting must apply regardless of the source of the sample and the patient's ability to pay ${ }^{17}$. Sometimes residual samples are often used without the patient's knowledge, which is quite unethical. However, there are many discussions and debates on who owns patient specimens and whether patients should share in profits, if financial gains are derived from leftover samples ${ }^{18,19}$. Rules and practices vary by region and institution. In current discussion, bio-banking of leftover specimens and the ethical issues associated with it are beyond its scope.

Conclusion: This paper is not intended to be a comprehensive one; however, we have tried to complement existing guidelines and documents that are available in some institutions and to offer a framework for addressing ethical issues encountered in the practice of clinical biochemistry and laboratory medicine in Bangladesh. As laboratory-based physicians, it is our utmost duty to uphold the standards of professionalism, be honest in all professional endeavors, and maintain a high level of personal integrity as well as maintain the existing professional code of ethics. We should also maintain a high level of quality in the product(s) of our professional endeavors, including validity and reliability of laboratory test results, interpretive opinions, 
scientific research, publication of data, and different forms of knowledge dissemination. Moreover, we should ensure promotion of safety and welfare of patients, employees, coworkers, colleagues, the public, and the environment.

\section{References:}

1. Taylor JR, Thompson PJ, Genzen JR, Hickner J, Marques MB. Opportunities to enhance laboratory professionals' role on the diagnostic team. Lab Med. 2017;48(1):97-103.

2. Wijeratne $\mathrm{N}$, Benatar SR. Ethical issues in laboratory medicine. J Clin Pathol. 2010;63(2):97-98.

3. Arora DR, Arora B. Ethics in laboratory medicine. Indian J Med Microbiol. 2007;25(3):179-180.

4. The Nuremberg Code (1947). BMJ 1996;313:1448.

5. Anonymous. World Medical Association Declaration of Geneva. Afr Health Sci. 2017;17(4):1203.

6. World Medical Association. World Medical Association Declaration of Helsinki: ethical principles for medical research involving human subjects. JAMA. 2013;310(20):2191-2194.

7. Department of Health, Education, and Welfare; National Commission for the Protection of Human Subjects of Biomedical and Behavioral Research. The Belmont Report: Ethical principles and guidelines for the protection of human subjects of research. J Am Coll Dent. 2014;81(3):4-13.

8. Sekadde-Kigondu CB, Higgins T, Bruns DE, Gronowski AM, Ethical considerations in clinical chemistry and laboratory medicine. (Available at: http://www.ifcc.org/media/446404/IFCC_TF_Et hics_in_Lab_Medicine.pdf) (Accessed on August 25, 2019).

9. Canadian Society for Medical Laboratory Science (CSMLS). CSMLS Code of Ethics. (Available at: https://www.csmls.org/About-Us/OurMembers/Code-of-Ethics.aspx) (Accessed on August 26, 2019).
10. Nyrhinen $T$, Leino-Kilpi $H$. Ethics in the laboratory examination of patients. J Med Ethics. 2000;26(1):54-60.

11. Leino-Kilpi H, Nyrhinen T, Katajisto J. Patients' rights in laboratory examinations: do they realize? Nurs Ethics. 1997;4:451-464.

12. Jefford M, Moore R. Improvement of informed consent and the quality of consent documents. Lancet Oncol. 2008;9:485-493.

13. Watwe JM. Disclosure of confidential medical information. Issues Med Ethics. 1998;6(2):56-57.

14. Borovecki A, Mlinaric A, Horvat M, Supak Smolcic V. Informed consent and ethics committee approval in laboratory medicine. Biochem Med (Zagreb). 2018;28(3):030201.

15. Plebani $M$. The detection and prevention of errors in laboratory medicine. Ann Clin Biochem. 2010;47(Pt 2):101-110.

16. Heher YK, Dintzis SM. Disclosure of harmful medical error to patients: a review with recommendations for pathologists. Adv Anat Pathol. 2018;25(2):124-130.

17. Stempsey WE. The virtuous pathologist - an ethical basis for laboratory medicine. Am J Clin Pathol. 1989;91(6):730-738.

18. Caenazzo L, Tozzo P, Pegoraro R. Biobanking research on oncological residual material: a framework between the rights of the individual and the interest of society. BMC Med Ethics. 2013;14:17.

19. Medical Research Council, UK. Human tissue and biological samples for use in research: operational and ethical guidelines. 2014. (Available at: https://mrc.ukri.org/publications/browse/humantissue-and-biological-samples-for-use-inresearch/) (Accessed on August 31, 2019).

Authors' contribution: M Mozaffor and S Parvin were involved in conception of the paper; M Mozaffor, M Tabassum, MT Sultan and S Parvin were equally involved in the literature search, manuscript writing and revision.

Conflict of interest: None to disclose. 